\title{
Formation of coherent rotational wavepack- ets in small molecule-helium clusters using impulsive alignment ${ }^{\dagger}$
}

\author{
Gediminas Galinis $^{a}$, Luis G. Mendoza Luna ${ }^{a}$, Mark J. Watkins ${ }^{a}$, \\ Andrew M. Ellis ${ }^{b}$, Russell S. Minns ${ }^{c}$, Mirjana Mladenović ${ }^{d}$, \\ Marius Lewerenz ${ }^{d}$, Richard Chapman ${ }^{e}$, I. C. Edmond Turcu ${ }^{e}$, \\ ${\text { Cephise } \text { Cacho }^{e}, \text { Emma Springate }}^{e}, \operatorname{Lev~Kazak}^{f}$, Sebastian Göde $^{f}$, \\ Robert Irsig $^{f}$, Slawomir Skruszewicz ${ }^{f}$, Josef Tiggesbäumker ${ }^{f}$, \\ Karl-Heinz Meiwes-Broer ${ }^{f}$, Arnaud Rouzée ${ }^{g}$, Jonathan G. Under- \\ wood $^{h}$, Marco Siano $^{i}$, Klaus von Haeften ${ }^{* a}$
}

Received Xth $X X X X X X X X X X 20 X X$, Accepted Xth $X X X X X X X X X 20 X X$

First published on the web $X$ th $X X X X X X X X X X 200 X$

DOI: $10.1039 / \mathbf{c 0 0 0 0 0 0 x}$

We show that rotational line spectra of molecular clusters with near zero permanent dipole moments can be observed using impulsive alignment. Aligned rotational wavepackets were generated by non-resonant interaction with intense femtosecond laser pump pulses and then probed using Coulomb explosion by a second, time-delayed femtosecond laser pulse. By means of a Fourier transform a rich spectrum of rotational eigenstates was derived. For the smallest cluster, $\mathrm{C}_{2} \mathrm{H}_{2}-\mathrm{He}$, we were able to establish essentially all rotational eigenstates up to the dissociation threshold on the basis of theoretical level predictions. The $\mathrm{C}_{2} \mathrm{H}_{2}-\mathrm{He}$ complex is found to exhibit distinct features of large amplitude motion and very early onset of free internal rotor energy level structure.

\section{Introduction}

Laser-induced alignment is an emerging technology for preparing molecules in well-defined quantum states, thereby aligning them with respect to the laser

${ }^{a}$ University of Leicester, Department of Physics \& Astronomy, Leicester, LE1 7RH, United Kingdom. Fax:+44 116252 2770; Tel: +44 116252 3525; E-mail: kvh6@le.ac.uk

${ }^{b}$ University of Leicester, Department of Chemistry, Leicester, LE1 7RH, United Kingdom

${ }^{c}$ University of Southampton, Department of Chemistry, Southampton, SO17 1BJ, United Kingdom

${ }^{d}$ Université Paris-Est, Laboratoire Modélisation et Simulation Multi Echelle, MSME UMR8208 CNRS, 5 bd Descartes, 77454 Marne-la-Vallée, France

${ }^{e}$ Central Laser Facility, Rutherford Appleton Laboratory, United Kingdom

${ }^{f}$ Institute for Physics, University of Rostock, Germany

${ }^{g}$ Max Born Institute, Berlin, Germany

${ }^{h}$ Department of Physics \& Astronomy, University College London,London WC1E 6BT

${ }^{i}$ Blackett Laboratory, Imperial College London, London SW7 2BW, United Kingdom

$\dagger$ Electronic Supplementary Information (ESI) available: [details of any supplementary information available should be included here]. See DOI: 10.1039/c000000x/ 
field ${ }^{1-8}$. When used with long laser pulse, the technique is often refereed as adiabatic alignment ${ }^{3-5,9}$, whereas for pulse duration shorter than the rotational period of the molecule, impulsive or field-free alignment is a common term. The impulsive alignment technique is derived from the well-known rotational coherence spectroscopy method that has been intensively applied to the investigation of the rotational structure of free molecules ${ }^{10-14}$, clusters ${ }^{15-17}$ and liquids ${ }^{18}$. Laser-induced impulsive alignment can be applied to all molecules with an anisotropy of polarisability. While initially, studies were performed in small linear molecules ${ }^{4,19,20}, 1 \mathrm{D}$ and 3D impulsive alignment of complex, asymmetric top molecules has been demonstrated ${ }^{21-25}$. Control over molecular alignment has enabled the investigation of processes such as strong field ionisation ${ }^{26,27}$, high harmonic generation ${ }^{28-30}$, gas-phase $\mathrm{X}$-ray ${ }^{31}$ and (photo-)electron diffraction experiments 31,32 .

In impulsive alignment an intense laser pulse interacts with molecules nonresonantly and populates rotational eigenstates via virtual states corresponding to the respective laser wavelength. The excitation pathway via a virtual state means that in impulsive alignment excitation of rotational levels requires two photons. The excitation of rotational states is equivalent to a Raman process with the associated selection rules for the parity and total angular momentum of the excited system. Depending on the duration, shape and intensity of the laser field the Raman process can induce sequential transitions, leading, for example, to excitation of high rotational levels. Likewise, rotational levels can be de-excited, or remain unchanged. The final state constitutes a wavepacket of rotational eigenstates. that periodically reassembles, representing alignment in space with respect to the laser polarisation axis. Following the propagation of the wavepacket in time provides dynamical information straightforwardly, such as the determination of coherence times. This is important for the investigation of time-dependent interactions, such as molecular collisions ${ }^{33}$. To illustrate the state of alignment in a classical picture, diatomics exposed to linearly polarised laser pulses will, for example, exhibit their figure axis aligned parallel and anti parallel with respect to the laser polarisation axis $(z)$. In quantum mechanical terms, alignment is equivalent to a population shift towards the highest positive and negative $M_{z}$ rotational states $^{8}$. If the amplitude of the excitation laser pulse decreases much faster than the rotational period of the molecule, then the rotational states remain populated and the wavepacket propagates in time and space even after the laser field has vanished ${ }^{7}$.

This paper is motivated by the prospect of adopting the impulsive alignment technique for elucidating the properties of free clusters. The investigation of free atomic and molecular clusters is an important pillar for understanding size effects and complexity in condensed matter ${ }^{34-37}$. Furthermore, this knowledge of intrinsic cluster properties is essential for the understanding how these clusters interact with their environment and is ultimately important for the applications of clusters in new materials ${ }^{38,39}$.

To generate clusters free of interactions, a supersonic expansion in vacuum is commonly employed: pressurised gas expanding through a nozzle into vacuum cools rapidly, thereby facilitating cluster growth, and forms a molecular beam, propagating through the vacuum apparatus ${ }^{40,41}$. A particular benefit of using molecular beams is that the cluster samples are continuously renewed, so that

2 | Faraday Discuss., [year], [vol], 1-25 This journal is $\odot$ The Royal Society of Chemistry [year] 
effects of any probing, such as radiation damage or fragmentation, does not affect subsequent measurements. An alternative possibility is to study clusters confined in ion traps ${ }^{42-45}$.

The propagating molecular beam can be probed by interaction with light, static electric or magnetic fields, by scattering from charged or neutral particles, or off space-fixed nanostructures. Thus, a variety of different techniques are currently available to study free clusters: laser spectroscopy ${ }^{46,47}$, ion depletion spectroscopy ${ }^{45}$, fluorescence spectroscopy ${ }^{48,49}$, mass spectrometry ${ }^{50-52}$, SternGerlach deflection ${ }^{53-55}$, static electric field deflection ${ }^{56-58}$, electron beam scattering ${ }^{59-61}$ and diffraction from space-fixed periodic nanostructures ${ }^{62,63}$. Very recently, time-resolved, single cluster diffraction using soft X-ray ${ }^{64}$ and hard Xrays ${ }^{65}$ has been employed.

Impulsive alignment is specifically beneficial for probing weakly bound clusters because spectral analysis is facilitated and enough information to benchmark physical models can be gained as we will illustrate here for small clusters of helium and a molecule. A characteristic feature of weakly bound molecular clusters is large amplitude motion. For such weakly bound clusters bending vibrations, internal rotations or other types of internal motion occur on similar time scales and therefore it is difficult, or often impossible to separate the wavefunction into distinct rotational and vibrational parts. Hence, a conventional rigid rotor-type Hamiltonian fails to describe their rotational level structure ${ }^{66}$. While pure rotational spectroscopy provides insight into entangled internal motions of molecular complexes and clusters, the experimental data reported in the literature is comparatively sparse. Furthermore, to form weakly bound clusters, very low temperatures have to be provided. This implies that only the lowest rotational levels are populated with the consequence that a conventional rotational spectrum may contain only few lines, or perhaps even just one - usually not enough for a comprehensive analysis of the structure, as pointed out by Nesbitt and Naaman ${ }^{67}$. By exciting high lying rotational levels in a wavepacket it is therefore possible to generate a rich rotational line spectrum despite the limitation of a cold initial thermal population.

Small helium clusters also attract considerable attention because they establish model systems by which the effect of size and complexity on quantum phenomena, such as superfluidity, can be studied. In the past twenty years molecules have been embedded in large helium droplets as well as in small helium clusters and their pure rotational and ro-vibrational frequency spectra have been studied using conventional microwave (MW) and infrared (IR) spectroscopy. In broad terms these studies have established that in ${ }^{4} \mathrm{He}$ droplets the molecules rotate almost unhindered, but with an increased apparent moment of inertia compared to the gas phase. Also, their spectral lines are broadened with respect to the gas phase, reflecting the interaction with the helium (see review articles for further details ${ }^{68-74}$ ). Although much progress has been made the processes leading to the increase of apparent moment of inertia and line broadening are not entirely understood. The availability of the complete pure rotational spectrum of small molecule-helium clusters will be an important complement to the previous and ongoing infrared and microwave-based spectral analysis of these systems and pave the way towards a comprehensive understanding of incipient superfluidity.

Here, we show the generation of rotational wavepackets of $\mathrm{C}_{2} \mathrm{H}_{2}-\mathrm{He}_{n}$ clus- 
ters using impulsive alignment. The phase of the wavepacket was detected as a function of time using Coulomb explosion. Fourier transformation produced a rich spectrum of discrete lines in the frequency domain that were attributed to rotational eigenstates of the clusters. A detailed analysis was carried out for the smallest complex, $\mathrm{C}_{2} \mathrm{H}_{2}-\mathrm{He}$. This weakly bound complex is an important model system that has previously been investigated using ab initio calculations ${ }^{75-78}$ and infrared spectroscopy in its deuterated form ${ }^{77}$, but whose pure rotational spectrum has not previously been reported. As part of the current study we also present an improved potential energy surface of this complex. A complete set of bound rovibrational levels for this surface was computed with a numerically exact discrete variable approach. The measured transitions of $\mathrm{C}_{2} \mathrm{H}_{2}-\mathrm{He}$ mapped practically all theoretically predicted transitions between bound rotational levels, showing excellent agreement between theory and experiment and demonstrating the successful application of the impulsive alignment method to obtain a complete rotational spectrum of a weakly bound complex. The knowledge of the complete level structure has prompted a more detailed theoretical analysis which revealed the importance of dynamical effects contributing to the pronounced freeinternal rotor behaviour of this complex.

While the demonstration of a rotational wavepacket-based spectral analysis is important for the field of cluster research our work gives directions in using $\mathrm{He}$ droplets for preparing and aligning exotic samples in the gas phase to perform diffraction measurements.

\section{Experimental details}

The experiments were conducted at the Rutherford Appleton Laboratory using the Artemis femtosecond laser beam line and the Atomic and Molecular Physics end-station, which was equipped with a source for the production of doped helium clusters in co-expansion. Apart from the cluster production the experiment was conceptually similar to that of Pentlehner $e t$ al. ${ }^{79}$, particularly the generation of rotational wavepackets and their detection using a pump-probe scheme. Briefly, $\mathrm{C}_{2} \mathrm{H}_{2}-\mathrm{He}_{n}$ clusters were generated in a supersonic expansion of $0.01 \%$ $\mathrm{C}_{2} \mathrm{H}_{2}$ diluted in $9 \mathrm{MPa} \mathrm{He}$ through a cooled pulsed valve of conical shape (half opening angle, $\alpha=20^{\circ}$ ) with a throttle diameter of $100 \mu \mathrm{m}$ (Even-Lavie noz$\mathrm{zle}^{80}$ ). The cluster beam propagated approximately $50 \mathrm{~mm}$ through the vacuum chamber into the focus of a velocity map imaging (VMI) detector ${ }^{81}$ where it was excited by two pulsed laser beams, both originating from a $30 \mathrm{fs}, 1 \mathrm{kHz}$ Ti:Sapphire laser operating at $800 \mathrm{~nm}$ (KM Labs Red Dragon). The laser system had two separate grating compressors, allowing the pulse durations of each laser beam to be independently controlled. The beams were co-linearly focused through a $f=500 \mathrm{~mm}$ lens into the molecular beam. The number of rotational levels in the wavepacket was controlled by adjusting the pulse length and intensity of the first laser pulse (the pump laser). The pump laser had a duration of $300 \mathrm{fs}$ and was operated across a range of intensities from $2 \times 10^{11} \mathrm{Wcm}^{-2}$ to $5 \times 10^{12} \mathrm{Wcm}^{-2}$, well below the level required to ionise the molecules. The probe pulse had a duration of $50 \mathrm{fs}$ and an intensity of $1 \times 10^{15} \mathrm{Wcm}^{-2}$, sufficient to instantly break molecular bonds in a Coulomb explosion, thereby generating $\mathrm{C}^{+}, \mathrm{C}^{2+}$ and $\mathrm{H}^{+}$fragment ions. The velocity vectors of the fragment ions, which

4| Faraday Discuss., [year], [vol], 1-25

This journal is $\odot$ The Royal Society of Chemistry [year] 
carried the molecular alignment information, were mass-selectively detected in a velocity map imaging (VMI) spectrometer ${ }^{81}$ whose detector plane was parallel to the polarisation plane of both laser beams ( $x y$ plane in Fig 1). Mass-selection was established by switching the gain of the microchannel plate detector so that it was only sensitive at the specific times when the ions of interest arrived at the detector. To extract alignment information the $\mathrm{C}^{+}$mass channel was chosen for detection.

The two-dimensional projection of the recoiling $\mathrm{C}^{+}$fragment directions and intensities was used to determine $I \times \cos ^{2} \theta$ for each position on the detector, where $\theta$ designates the angle between the polarisation of the pump laser and the projected velocity vector and $I$ the intensity. The origin of this vector was set to the centre of the image defined by the centre of mass of the ion distribution. The end of the vector pointed to a pixel representing the intensity of ions at this position. The average over the entire detector area, $\left\langle\cos ^{2} \theta_{2 D}\right\rangle$, is proportional to the molecular alignment $\left\langle\cos ^{2} \theta_{3 D}\right\rangle^{79,82}$. The quantity $\left\langle\cos ^{2} \theta_{3 D}\right\rangle$, indicating the phase of the rotational wavefunction, is a true quantum mechanical observable whose exact determination would require the projected velocity map images to be inverted, using an inverse Abel transformation ${ }^{83-85}$. Since the recorded ion distributions did not exhibit spherical symmetry the inversion procedure is technically challenging. The parameter $\left\langle\cos ^{2} \theta_{2 D}\right\rangle$ was determined instead as a function of time by scanning the delay between the pump and probe laser pulses to reveal the rotational dynamics of the clusters. Because of the proportionality between $\left\langle\cos ^{2} \theta_{3 D}\right\rangle$ and $\left\langle\cos ^{2} \theta_{2 D}\right\rangle$ this lack of spherically symmetric images had no consequences for the derivation of spectroscopic information and the conclusions of our experimental results.

\section{Results}

\subsection{Coulomb explosion}

Fig. 2 shows raw velocity map images recorded selectively for the $\mathrm{C}^{+}$fragment under different expansion conditions. These raw images show ion fragment intensities with an angular and radial distribution that illustrate the Coulomb explosion process. Dashed rings have been introduced to indicate the centre position and to highlight differences in the radial distribution. Distinct changes in the Coulomb explosion pattern are observed for varying expansion conditions: panel (a) in Fig. 2 shows the ion image for expansions where the nozzle was held at a temperature of $293 \mathrm{~K}$ and panel (b) shows the image obtained when the nozzle was cooled to $203 \mathrm{~K}$ to facilitate the formation of clusters of $\mathrm{C}_{2} \mathrm{H}_{2}$ with helium. The ion image in (a) shows relatively sharp features, with evidence of more than one fragmentation channel leading to the formation of $\mathrm{C}^{+}$. These features were attributed to unbound $\mathrm{C}_{2} \mathrm{H}_{2}$ as the dominant species. The angular distributions of the two images are identical and largely influenced by the anisotropic laser ionisation. However, the radial distributions differ: panel (b) shows considerably higher kinetic energies than (a). The increased kinetic energy can be taken as an indicator for the generation of further charges, even though the laser parameters themselves have not changed. The temperature variation from 293 to $203 \mathrm{~K}$ increases the gas number density in the interaction region by $30 \%$, which cor-

This journal is $\odot$ The Royal Society of Chemistry [year]

Faraday Discuss., [year], [vol], 1-25|5 


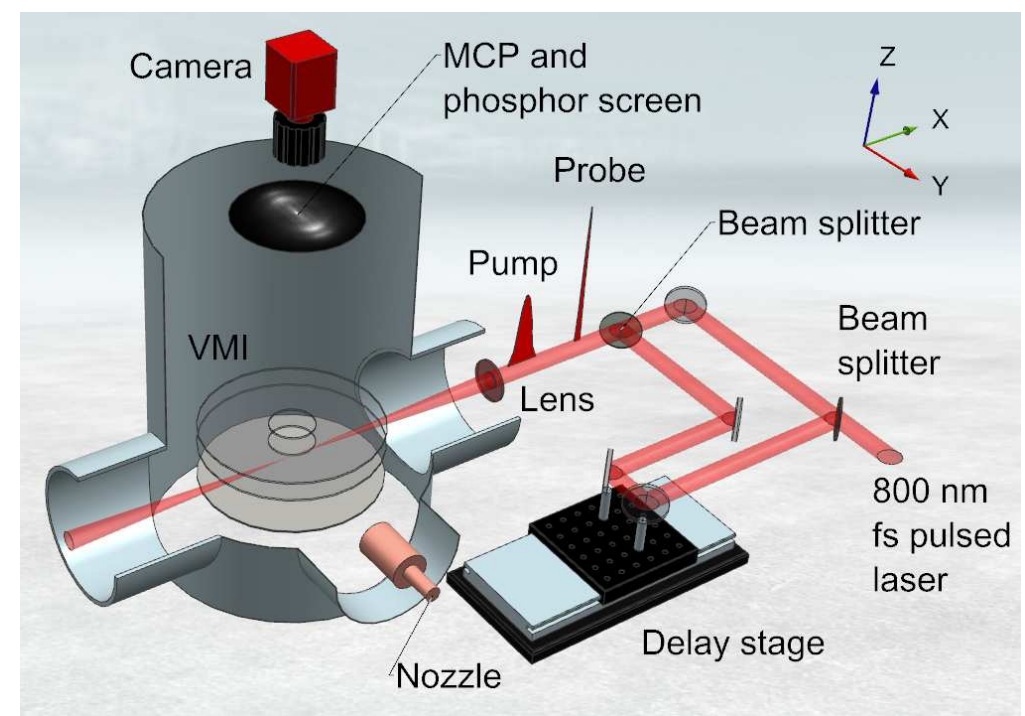

Fig. 1 Schematics of the experimental setup comprising a molecular beam machine, a laser system and a velocity map imaging detector coupled to a CCD camera. Clusters and molecules prepared in the molecular beam were impulsively aligned and probed using a laser pump-probe scheme, where the delay between the two laser pulses is generated with a movable stage. The state of alignment was probed for time delays of up to $600 \mathrm{ps}$, corresponding to the maximum displacement of the delay stage.

responds to a reduction of the internuclear separation of particles within the jet of $11 \%$. This small change means that space charge effects do not explain the increase of ion velocities observed in Fig. 2. It is more likely that the processes responsible occur within the clusters because of the much higher atomic number density than within the unclustered jet. Further charges can be generated within the $\mathrm{C}_{2} \mathrm{H}_{2}$ molecules, but also $\mathrm{He}$ atoms could be ionised ${ }^{86}$. A second possibility is that the higher kinetic energies originate from inelastic scattering of $\mathrm{C}^{2+}$ fragments or even higher charged fragments when leaving the cluster. Subsequently, these highly charged ions recombine with electrons, producing $\mathrm{C}^{+}$fragments.

\subsection{Time-resolved alignment and Fourier transformation}

The time-resolved molecular alignment parameter, $\left\langle\cos ^{2}(\theta)_{2 D}\right\rangle(\mathrm{t})$, is shown in Fig. 3(a) for a time period of $16 \mathrm{ps}$ and for conditions favouring cluster formation similar to Fig. 2(b). The strongest features consist of a pattern of six oscillations which reappear every rotational period $\tau$. This pattern matches the time-resolved alignment spectrum expected for free, cold $\mathrm{C}_{2} \mathrm{H}_{2}$ molecules. The features extend over the full range of the scan up to 600 ps, as shown in Fig. 3(b). No damping in amplitude is observed, so one can infer that the coherence of the rotational wavepacket is at least $600 \mathrm{ps}$. Revivals of small acetylene-helium clusters $\left(\mathrm{C}_{2} \mathrm{H}_{2}-\mathrm{He}_{n}\right)$ are difficult to directly identify in the alignment scan because of the dominant signals from free acetylene rotation. A much clearer picture is obtained when a discrete Fourier transform is performed, producing a rotational

6 | Faraday Discuss., [year], [vol], 1-25

This journal is $\odot$ The Royal Society of Chemistry [year] 

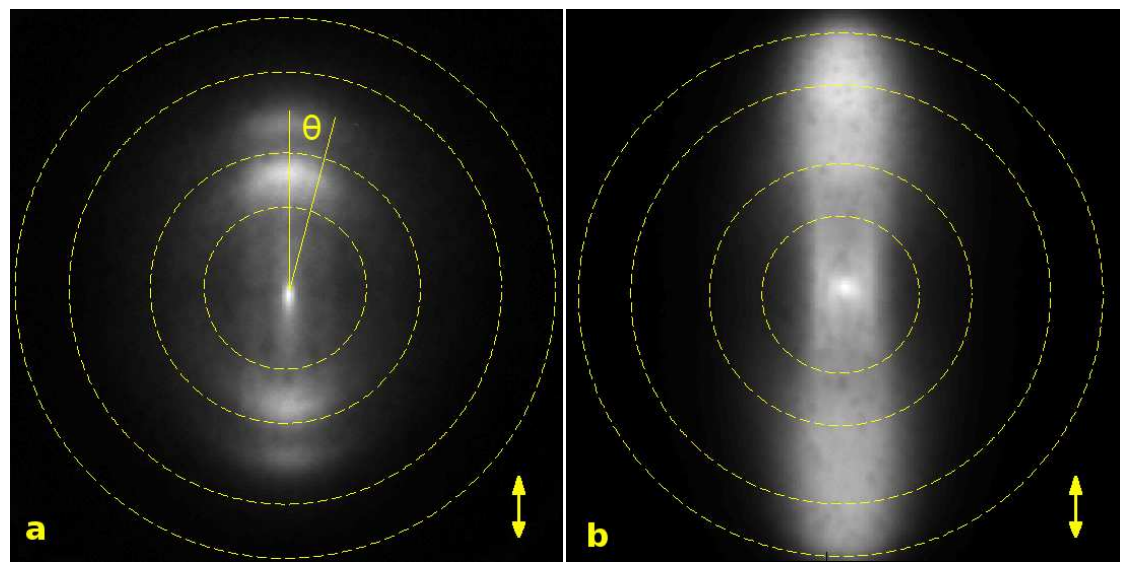

Fig. 2 Velocity map images of $\mathrm{C}^{+}$fragments generated via Coulomb explosion. (a) was recorded at nozzle temperatures of $293 \mathrm{~K}$ and shows fragments originating from free $\mathrm{C}_{2} \mathrm{H}_{2}$. In (b) the nozzle temperature was $203 \mathrm{~K}$, facilitating cluster formation, but otherwise identical conditions. (b) shows increased kinetic energy compared to (a). The yellow double-headed arrow indicates the direction of the pump and probe laser polarisations, which are parallel to the $y$-axis in Fig. 1. The dashed circles are guides to the eye.

frequency spectrum ${ }^{87}$.

The discrete Fourier transform, $F_{\omega}$, is defined by

$$
F_{\omega}=\sum_{t=0}^{N-1} f_{t} e^{-2 \pi i \omega t / N}
$$

where $N$ designates the number of data points being transformed, $f_{t}$ is the $t^{\text {th }}$ element in the time domain and $\omega$ is a variable in the frequency domain. The $N$ complex numbers derived from equation (1) were converted into a power spectrum by adding the squared real and imaginary parts of $F_{\omega}$ :

$$
\operatorname{Re}\left(F_{\omega}\right)^{2}+\operatorname{Im}\left(F_{\omega}\right)^{2}
$$

The resulting power spectrum is shown in Fig. 4.

A series of discrete lines in Fig. 4 corresponds to particular frequency contributions of $\mathrm{C}_{2} \mathrm{H}_{2}$ to the rotational wavepackets. The observed lines were recorded at a pump laser intensity of $2.5 \times 10^{11} \mathrm{Wcm}^{-2}$ and coincide with the beat frequencies of free $\mathrm{C}_{2} \mathrm{H}_{2}$ at $6 b+4 n b$, where $b$ is the $\mathrm{C}_{2} \mathrm{H}_{2}$ rotational constant ${ }^{\dagger}$ $\left(b=1.1767 \mathrm{~cm}^{-1}\right)^{88}$ and $n=0,1,2,3$. The first line at $6 b$ frequency is equivalent to the $J^{\prime}=2 \leftarrow J^{\prime \prime}=0$ transition, with successive lines at $10 b, 14 b, 18 b$. The significantly higher intensity of the contribution from the $J=1$ state compared with the $J=0$ state is attributed to nuclear spin statistics describing the occupation ratio of even $J$ (para):odd $J$ (ortho) levels, which in this case is $1: 3^{89}$. Since

$\dagger B$ conventionally denotes the rotation constant of a linear molecule. However, in this context we want to differentiate between the internal rotation of the molecule within a complex, later in the text denoted as $b$, and the overall rotational constant of the complex, as $B$. 


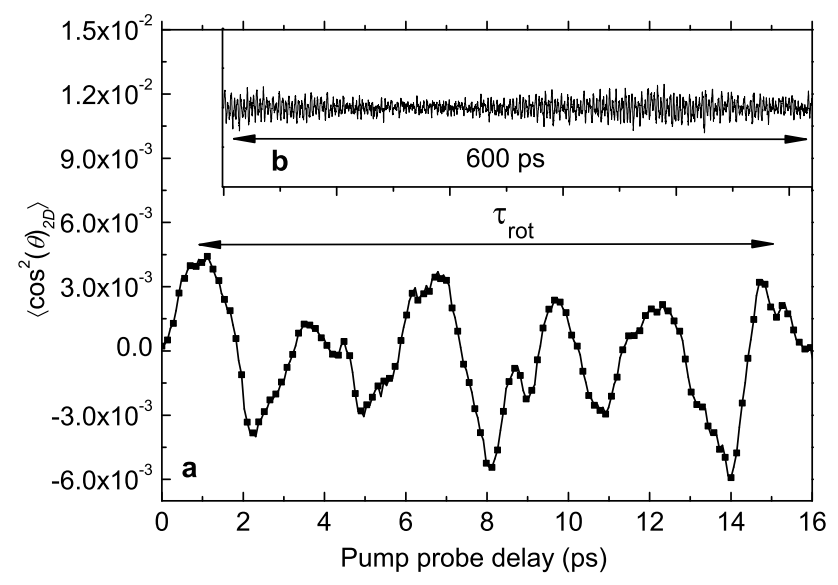

Fig. 3 Time-resolved alignment of $\mathrm{C}_{2} \mathrm{H}_{2}$ co-expanded with helium. (a) Rotational revivals obtained under conditions where the helium stagnation pressure and temperature were $9 \mathrm{MPa}$ and $212 \mathrm{~K}$, respectively. Note that the baseline has been subtracted and the data have been smoothed. The time difference between two full revivals, indicated by a double-headed arrow, is equal to the rotational period of acetylene and inversely

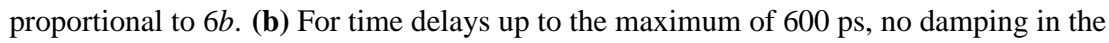
amplitude was observed. The coherence time of the wavepacket for free $\mathrm{C}_{2} \mathrm{H}_{2}$ molecules is therefore at least $600 \mathrm{ps}$.

Fig. 4 displays the power spectrum, the ratio in principle should be 1:9. However, when additional $J$-dependent coupling terms for two-photon transitions are taken into account a revised ratio of 6.25 is expected. This expected ratio is in satisfactory agreement with the observed relative peak intensities.

A much weaker series of discrete lines is seen at $4 b, 8 b$ and $12 b$, notably around 9.2 and $14.2 \mathrm{~cm}^{-1}$. The position of these lines can be expressed through the series $2 b+2 n b$, for $n \geq 1$, with every second line overlapping with the Raman allowed transitions at $6 b+4 n b$. The occurrence of these lines does not comply with the $\Delta J=0, \pm 2$ selection rule for Raman transitions of diatomic molecules. We note that the $\Delta J=0, \pm 2$ selection rule would be relaxed for symmetric top molecules and may indeed indicate the involvement of $\mathrm{C}_{2} \mathrm{H}_{2}-(\mathrm{He})_{n}$ clusters. However, the good match of these transitions with multiples of the $b$ constant of free $\mathrm{C}_{2} \mathrm{H}_{2}$ is difficult to explain considering that in other molecule-helium complexes such lines are always shifted ${ }^{90}$.

\section{Assignment of $\mathrm{C}_{2} \mathrm{H}_{2}$-He lines: statistical analysis and theo- retical predictions}

In the low frequency range in Fig. 4(c) numerous peaks are observed which have frequencies well below those of free $\mathrm{C}_{2} \mathrm{H}_{2}$. These lines are attributed to clusters because they correspond to a larger moment of inertia than is possible for free $\mathrm{C}_{2} \mathrm{H}_{2}{ }^{90,91}$. To fully assess our assignment in this frequency range and to account for the low intensities a careful statistical analysis of the noise was performed. A region of the spectrum between 100 to $240 \mathrm{~cm}^{-1}$ which was free free of molec-

8 | Faraday Discuss., [year], [vol], 1-25

This journal is $\odot$ The Royal Society of Chemistry [year] 


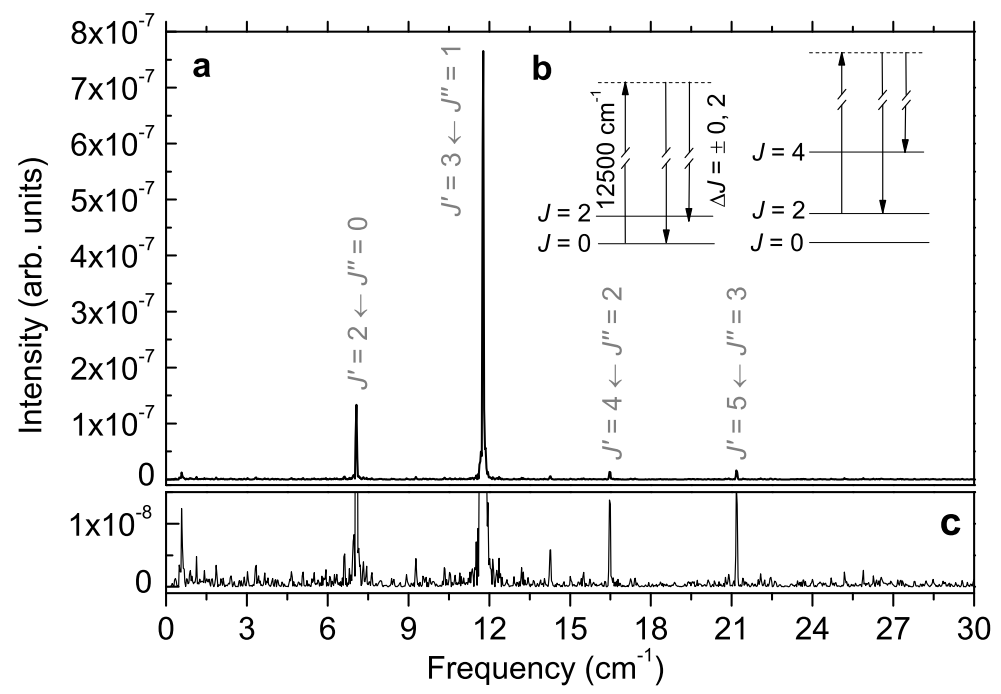

Fig. 4 Overview spectra. (a) Power spectrum of the time-resolved molecular alignment. The power spectra are dominated by strong peaks arising from beats between rotational levels of free $\mathrm{C}_{2} \mathrm{H}_{2}$ connected by $\Delta J=0, \pm 2$. (b) The rotational levels in the wavepacket are excited through sequential Raman excitations with an $800 \mathrm{~nm}\left(12,500 \mathrm{~cm}^{-1}\right)$ laser pulse. This process sequentially populates higher levels via virtual states, as schematically illustrated in the inset. Panel (c) shows an expanded view of the power spectrum revealing numerous weak peaks.

ular frequencies was identified as suitable for a quantitative determination of the confidence level of our data and analysed to assess whether the intensity variation is stochastic. The signal height distribution was then investigated and the level at which a random event could be excluded with $99.9 \%$ certainty was determined. Details of the noise analysis are provided in the online-supplementary information.

While this assessment of the noise level revealed a large number of lines exceeding the confidence level, focus was placed on the detailed analysis of the smallest complex, the $\mathrm{C}_{2} \mathrm{H}_{2}-\mathrm{He}$ complex. For this complex a potential energy surface and the energies of rotational states were calculated to guide the assignment. The predicted energy levels were then compared with the line positions observed in the spectrum.

\subsection{Coherence times of $\mathrm{C}_{2} \mathrm{H}_{2}-(\mathrm{He})_{n}$}

An enlarged view of the low frequency region, including a dashed line to indicate the $99.9 \%$ confidence level, is shown in Fig. 5. A large number of lines, attributed to $\mathrm{C}_{2} \mathrm{H}_{2}-\mathrm{He}_{n}$ clusters and with intensities above the confidence level, are observed. The full widths at half maximum of these lines are $0.03 \mathrm{~cm}^{-1}$, which matches the experimental limit in resolution set by the total time delay of 600 ps. Hence, the width of the lines assigned to $\mathrm{C}_{2} \mathrm{H}_{2}-\mathrm{He}_{n}$ clusters is consistent with a coherence time for the rotational wavepacket of at least 600 ps. We note that such long coherence times are not unexpected for small molecu- 


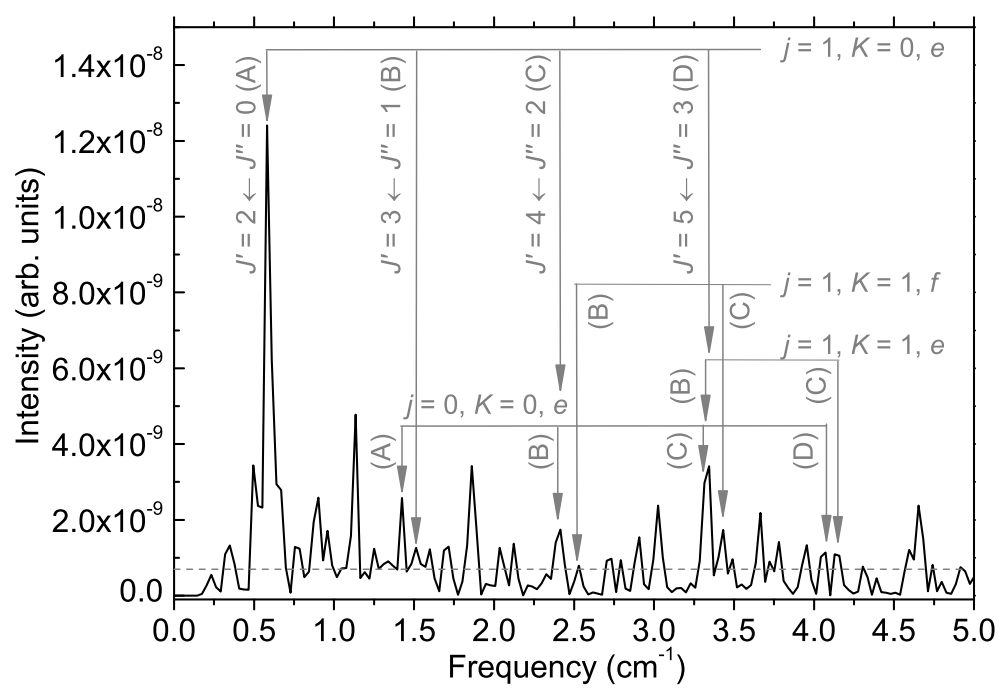

Fig. $5 \mathrm{C}_{2} \mathrm{H}_{2}-\mathrm{He}$ spectrum in the frequency region below $5 \mathrm{~cm}^{-1}$. The $99.9 \%$ confidence level, established through the second order $\chi^{2}$ spectral distribution of the noise, is indicated by a horizontal dashed line. The spectral lines are labelled in alphabetical order with respect to the total angular momentum of the initial state. See text and the energy diagram in Fig. 8 for further information.

lar clusters, given that predissociation or other events that destroy coherence are very unlikely on a time scale of sub-nanoseconds. This finding further conforms with the spectroscopic work in the frequency-domain, which has established that molecules residing inside superfluid ${ }^{4} \mathrm{He}$ droplets rotate almost freely ${ }^{92}$. Interestingly, a recent study by Pentlehner et al. ${ }^{79}$ showed rapid loss of coherence for non-adiabatic alignment of $\mathrm{CH}_{3} \mathrm{I}$ in helium droplets, i.e. within a single rotational period. This observation contradicts our results for $\mathrm{C}_{2} \mathrm{H}_{2}-\mathrm{He}$ and the many frequency-domain studies of molecules in helium droplets. This discrepancy is currently unexplained.

As detailed below we can assign some of the $\mathrm{C}_{2} \mathrm{H}_{2}-\mathrm{He}_{n}$ features to the $\mathrm{C}_{2} \mathrm{H}_{2}-$ He complex. The other peaks in Fig. 5 are attributed to larger $\mathrm{C}_{2} \mathrm{H}_{2}-\mathrm{He}_{n}(n \geq 2)$ complexes. A detailed assignment is difficult due to a lack of models for weakly bound clusters consisting of more than one helium atom. Our assignment of the $n=1$ case is guided by theory, as described in the next section.

\subsection{Electronic structure calculations}

Total electronic energies for $\mathrm{C}_{2} \mathrm{H}_{2}$ and $\mathrm{C}_{2} \mathrm{H}_{2}$-He were computed with the coupled cluster method with single and double substitutions and perturbative treatment of triples, $\operatorname{CCSD}(\mathrm{T})$, and all electrons were included in the correlation treatment. Core-optimised correlation consistent augmented (doubly augmented for $\mathrm{He}$ ) basis sets, (d)aug-cc-pCVXZ, X=T,Q,5,6, as developed by Dunning and coworkers $^{93,94}$, were employed as implemented in the MOLPRO electronic structure package $^{95}$. Geometry optimisations carried out for the complex and isolated 


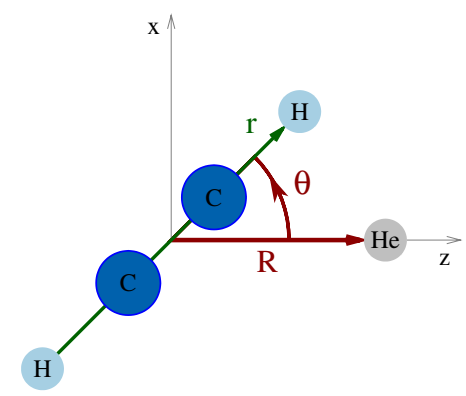

Fig. 6 Jacobi coordinates for the complex $\mathrm{C}_{2} \mathrm{H}_{2}$-He. The body-fixed $z$-axis is aligned with the Jacobi vector $\mathbf{R}$.

$\mathrm{C}_{2} \mathrm{H}_{2}$ with basis set sizes up to sextuple zeta quality show that complexation with $\mathrm{He}$ changes the geometry of $\mathrm{C}_{2} \mathrm{H}_{2}$ at most by $0.0001 \AA$. The $\mathrm{C}_{2} \mathrm{H}_{2}$ unit was consequently fixed at its experimental ground state expectation geometry, ${ }^{88}$ $r_{0}(\mathrm{CC})=1.20830 \AA$ and $r_{0}(\mathrm{CH})=1.05756 \AA$.

The interaction between $\mathrm{C}_{2} \mathrm{H}_{2}$ and a helium atom was explored in a Jacobi coordinate system with a Jacobi vector $\mathbf{R}$ pointing from the centre of mass of $\mathrm{C}_{2} \mathrm{H}_{2}$ to the helium atom and a Jacobi angle $\theta$ enclosed between $\mathbf{R}$ and the $\mathrm{C}_{2} \mathrm{H}_{2}$ molecular axis, as shown in Fig. 6. We used a grid of 300 points covering $0 \leq \theta \leq 90^{\circ}$ in steps of $10^{\circ}$ and radial grids which were optimised at each value of $\theta$, typically ranging between 2.50 and $20 \AA$. Total energies for the complex obtained with basis sets from triple zeta, (d)aug-cc-pCVTZ, to quintuple zeta, (d)aug-cc-pCV5Z, level were extrapolated to the complete basis set (CBS) limit using the procedure of Peterson et al. ${ }^{96,97}$, which turned out to give more consistent results than the more common procedure of Helgaker and coworkers. ${ }^{98}$ Interaction energies were determined from the estimated CBS energies by subtracting the result of an extrapolation for essentially separated monomers at $R=100 \AA$ and $\theta=0$. The grid point with the strongest interaction is at $R=4.32 \AA$ and $\theta=0$ with a potential value of $V_{\text {int }}=-25.100 \mathrm{~cm}^{-1}$ relative to separate monomers at $V=0$. This is noticeably below the previous best estimate of $-24.21 \mathrm{~cm}^{-1} .{ }^{99}$ The rotational constant of the complex at its electronic equilibrium geometry is found to be $B_{e}=0.2119 \mathrm{~cm}^{-1}$ and the corresponding permanent dipole moment is $0.029 \mathrm{D}$. The spherically averaged dipole polarisability exhibits a very weak dependence on the complex geometry and has a value of $\bar{\alpha}=24.0 a_{0}^{3}$ which is essentially the sum of the monomer values of $22.6 a_{0}^{3}$ and $1.4 a_{0}^{3}$ for $\mathrm{C}_{2} \mathrm{H}_{2}$ and $\mathrm{He}$, respectively, providing further indication of the very weak van der Waals interaction.

\subsection{Analytical representation of the interaction potential}

The interaction between $\mathrm{C}_{2} \mathrm{H}_{2}$ and a helium atom contains dispersion and induction contributions, where the latter arise from the quadrupole moment of $\mathrm{C}_{2} \mathrm{H}_{2}$. The asymptotic $R$-dependence should therefore contain only even inverse powers of $R$. We adopted an angle-dependent extended Tang-Toennies ${ }^{100}$ form to 
represent the ab initio interaction energies:

$$
\begin{aligned}
V_{\text {int }}(R, \theta)= & A(\theta) \exp \left\{-\left(b_{1}(\theta) R+b_{2}(\theta) R^{2}\right)\right\} \\
& -\sum_{k=3,8} f_{2 k}(b(\theta), R) \frac{C_{2 k}(\theta)}{R^{2 k}}
\end{aligned}
$$

The radial parameters $X=A, b_{1}, b_{2}, C_{6}, C_{8}, C_{10}$ are expanded over even order Legendre polynomials according to

$$
X(\theta)=\sum_{l=0,2, \ldots} X^{(l)} P_{l}(\theta)
$$

Higher order coefficients $C_{2 k}, k>5$, are defined by the standard recursion $C_{2 k+2}=$ $\left(C_{2 k} / C_{2 k-2}\right)^{3} C_{2 k-4}$ and the functions $f_{2 k}$ are Tang-Toennies damping functions with $b(\theta)=b_{1}(\theta)+2 b_{2}(\theta) R$. The present fitting model includes terms up to $l=6$ and contains 24 free parameters which were adjusted by a non-linear least squares procedure. ${ }^{101}$ This reproduces the 235 ab initio interaction energies falling below $+200 \mathrm{~cm}^{-1}$ with a root mean square error of $0.039 \mathrm{~cm}^{-1}$ and a largest deviation of $0.22 \mathrm{~cm}^{-1}$. The coefficients for this analytical representation are given in atomic units in Table. 1. Figure 7(a) displays the interaction potential with its minimum at linear $\mathrm{C}_{2} \mathrm{H}_{2}-\mathrm{He}$ and a saddle point at the $\mathrm{T}$-shaped arrangement.

Table 1 Coefficients for the analytical representation of the $\mathrm{C}_{2} \mathrm{H}_{2}-\mathrm{He}$ interaction potential, equations (3) and (4) (all values in atomic units).

\begin{tabular}{rrrr}
\hline$A^{(0)}$ & $0.1918812 \mathrm{E}+02$ & $C_{6}^{(0)}$ & $0.1248242 \mathrm{E}+02$ \\
$A^{(2)}$ & $0.2504029 \mathrm{E}+02$ & $C_{6}^{(2)}$ & $-0.2662071 \mathrm{E}+01$ \\
$A^{(4)}$ & $0.8700405 \mathrm{E}+01$ & $C_{6}^{(4)}$ & $-0.4200783 \mathrm{E}+01$ \\
$A^{(6)}$ & $0.1256556 \mathrm{E}+01$ & $C_{6}^{(6)}$ & $-0.5564107 \mathrm{E}+01$ \\
$b_{1}^{(0)}$ & $0.1327564 \mathrm{E}+01$ & $C_{8}^{(0)}$ & $0.1318068 \mathrm{E}+04$ \\
$b_{1}^{(2)}$ & $-0.3394156 \mathrm{E}+00$ & $C_{8}^{(2)}$ & $0.1819554 \mathrm{E}+04$ \\
$b_{1}^{(4)}$ & $-0.1268924 \mathrm{E}+00$ & $C_{8}^{(4)}$ & $0.8531615 \mathrm{E}+03$ \\
$b_{1}^{(6)}$ & $0.1446208 \mathrm{E}-01$ & $C_{8}^{(6)}$ & $0.1011623 \mathrm{E}+04$ \\
$b_{2}^{(0)}$ & $0.4538917 \mathrm{E}-01$ & $C_{10}^{(0)}$ & $-0.2223242 \mathrm{E}+05$ \\
$b_{2}^{(2)}$ & $0.4238423 \mathrm{E}-01$ & $C_{10}^{(2)}$ & $-0.2251989 \mathrm{E}+05$ \\
$b_{2}^{(4)}$ & $0.1040915 \mathrm{E}-01$ & $C_{10}^{(4)}$ & $-0.1157502 \mathrm{E}+05$ \\
$b_{2}^{(6)}$ & $-0.1945577 \mathrm{E}-02$ & $C_{10}^{(6)}$ & $-0.3578250 \mathrm{E}+05$ \\
\hline
\end{tabular}

\subsection{Rovibrational calculations}

Bound rovibrational levels for the fitted surface were calculated with the DVRDGB method, which uses a discrete variable representation (DVR) for the angular coordinate and a distributed Gaussian basis (DGB) for the radial degree of freedom ${ }^{102}$. This method uses an exact kinetic energy operator and makes no approximations aside from the Born-Oppenheimer concept of a potential energy surface, whose accuracy is only limited by the quality of the electronic structure treatment. We used atomic masses in conjunction with 51 Gauss-Legendre DVR

12 | Faraday Discuss., [year], [vol], 1-25

This journal is @ The Royal Society of Chemistry [year] 

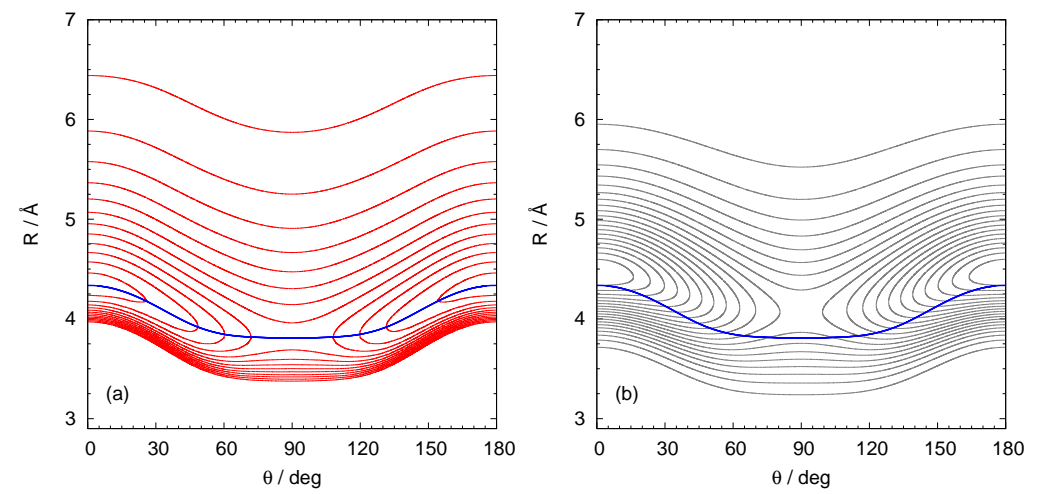

Fig. 7 (a) Contour plot of the $\mathrm{C}_{2} \mathrm{H}_{2}-\mathrm{He}$ interaction potential. The contour interval is $2 \mathrm{~cm}^{-1}$ and the lowest contour is at $V_{\text {int }}=-24 \mathrm{~cm}^{-1}$. (b) Contour plot of the $\left|\Psi_{0}\right|^{2}$ probability density of the ground state of $\mathrm{C}_{2} \mathrm{H}_{2}-\mathrm{He}$. Contours are given at intervals of $5 \%$ of the maximum value. For both figures, the blue curve indicates the minimum energy path.

points in $\theta$ and an angle dependent radial basis composed of up to 85 non-evenly distributed Gaussians between $4 a_{0}$ and $300 a_{0}$. Energy eigenvalues and wave functions were computed for both parities ( $p=0,1$ corresponding to even and odd parity states, respectively) and for total angular momentum $0 \leq J \leq 10$, but only levels up to $J=5$ were found to be bound. Energy levels are converged to better than $0.001 \mathrm{~cm}^{-1}$. The computed rovibrational ground state energy is $-7.417 \mathrm{~cm}^{-1}$. The square, $\left|\Psi_{0}\right|^{2}$, of the corresponding ground state $J=0$ wave function is displayed in Fig. 7(b). Maxima are visible for the two equivalent linear minimum energy arrangements but the probability density is clearly spread over the entire angular domain and remains above $40 \%$ of its maximum even at the saddle point at $\theta=90^{\circ}$. At all angles the pronounced anharmonicity shifts the radial position of the $\left|\Psi_{0}\right|^{2}$ maximum significantly outward with respect to the minimum energy path. The impact of this strong delocalisation on the rovibrational level structure will be described in more detail in the Discussion section.

\subsection{Assignment of experimentally observed lines}

The assignment of the peaks in Fig. 5 was made using the transitions frequencies predicted from the theoretical model described above. In particular, we looked for direct coincidence between the theory and experiment within experimental errors $\left(0.03 \mathrm{~cm}^{-1}\right)$. The theoretical prediction for the first excited state, the intermolecular stretching vibration, is $7.415 \mathrm{~cm}^{-1}$. Our spectrum shows a signal at a matching energy but the marginal nature of this state, only $0.002 \mathrm{~cm}^{-1}$ below the dissociation threshold and at the limits of the theoretical treatment does not allow a firm assignment without further work. The expected Raman transitions for $\mathrm{C}_{2} \mathrm{H}_{2}-\mathrm{He}$ are marked in Fig. 8 by vertical arrows and text, showing the measured energy differences in $\mathrm{cm}^{-1}$. The energy levels are labelled using the quantum numbers $j, J, K$ and parity labels $e$ and $f^{77}$. Note that only the total angular momentum quantum number $J$ and the parity of the wave functions are rigorous quantum numbers. The $j$ quantum number refers to the internal rotation 
Table 2 Energy level assignment of measured lines. Quantum numbers of the initial states are denoted by $j^{\prime \prime}, K^{\prime \prime}, J^{\prime \prime}$ and of the final states by $j^{\prime}, K^{\prime}, J^{\prime}$. The experimental line positions are given in $\mathrm{cm}^{-1}$ and the estimated error margin in each case is $\pm 0.03 \mathrm{~cm}^{-1}$.

\begin{tabular}{ccccccccc} 
Sym. & $j^{\prime \prime}$ & $K^{\prime \prime}$ & $J^{\prime \prime}$ & $j^{\prime}$ & $K^{\prime}$ & $J^{\prime}$ & Exp. & Calc. \\
\hline$e$ & 0 & 0 & 0 & 0 & 0 & 2 & 1.43 & 1.46 \\
$e$ & 0 & 0 & 1 & 0 & 0 & 3 & 2.41 & 2.40 \\
$e$ & 0 & 0 & 2 & 0 & 0 & 4 & 3.31 & 3.28 \\
$e$ & 0 & 0 & 3 & 0 & 0 & 5 & 4.07 & 4.08 \\
$e$ & 1 & 0 & 0 & 1 & 0 & 2 & 0.58 & 0.60 \\
$e$ & 1 & 0 & 1 & 1 & 0 & 3 & 1.51 & 1.49 \\
$e$ & 1 & 0 & 2 & 1 & 0 & 4 & 2.41 & 2.41 \\
$e$ & 1 & 0 & 3 & 1 & 0 & 5 & 3.30 & 3.31 \\
$e$ & 1 & 1 & 1 & 1 & 1 & 3 & 3.31 & 3.29 \\
$e$ & 1 & 1 & 2 & 1 & 1 & 4 & 4.16 & 4.15 \\
$f$ & 1 & 1 & 1 & 1 & 1 & 3 & 2.53 & 2.50 \\
$f$ & 1 & 1 & 2 & 1 & 1 & 4 & 3.43 & 3.43
\end{tabular}

of the $\mathrm{C}_{2} \mathrm{H}_{2}$ unit and $K$ is the projection of $J$ onto the intermolecular axis (see Fig. 6). Although approximate, these alternative quantum numbers turn out to be useful to describe the energy level pattern. The present calculation provides a better match to the measured line positions than the best potential energy surface available prior to this work ${ }^{78}$. The assignment of the lines is also shown in Table 2.

\section{Discussion}

\subsection{Rotational level pumping}

The identified lines of $\mathrm{C}_{2} \mathrm{H}_{2}$-He constitute a rich spectrum showing the possibility of sequential rotational energy level pumping. For example, the lower levels in the $J^{\prime}=4 \leftarrow J^{\prime \prime}=2$ and the $J^{\prime}=5 \leftarrow J^{\prime \prime}=3$ transitions in the $j=0, K=0, e$ manifold, the $J^{\prime}=4 \leftarrow J^{\prime \prime}=2$ and $J^{\prime}=5 \leftarrow J^{\prime \prime}=3$ transitions in the $j=1, K=0$, $e$ manifold, as well as the $J^{\prime}=4 \leftarrow J^{\prime \prime}=2$ transitions in the $j=1, K=1, e$ and $f$ manifolds, have presumably gained population through Raman transitions from lower levels. We note that increased pump laser power does not increase the intensity of these lines in a straightforward fashion. At the highest pump intensity of $5 \times 10^{12} \mathrm{Wcm}^{-2}$ the $J^{\prime}=1 \leftarrow J^{\prime \prime}=1$ transitions in the $j=1, K=1, f$ manifold becomes the strongest line. This transition also remains stronger than all other $\mathrm{C}_{2} \mathrm{H}_{2}-\mathrm{He}_{n}$ lines when the expansion conditions are changed to promote clusters comprising more than one helium atom. More experimental work is needed to confirm this observation and to elucidate details of these trends.

The highest rotational level populated lies beyond the dissociation threshold for $\mathrm{C}_{2} \mathrm{H}_{2}-\mathrm{He}$ when internal rotation is not excited $(j=0)$. This level, which belongs to the $j=1, K=1$, e manifold, can predissociate provided a pathway for de-excitation of the internal rotation of the $\mathrm{C}_{2} \mathrm{H}_{2}$ molecule exists.

The observation of this energy level on the timescale of this experiment,

14 | Faraday Discuss., [year], [vol], 1-25

This journal is $\odot$ The Royal Society of Chemistry [year] 


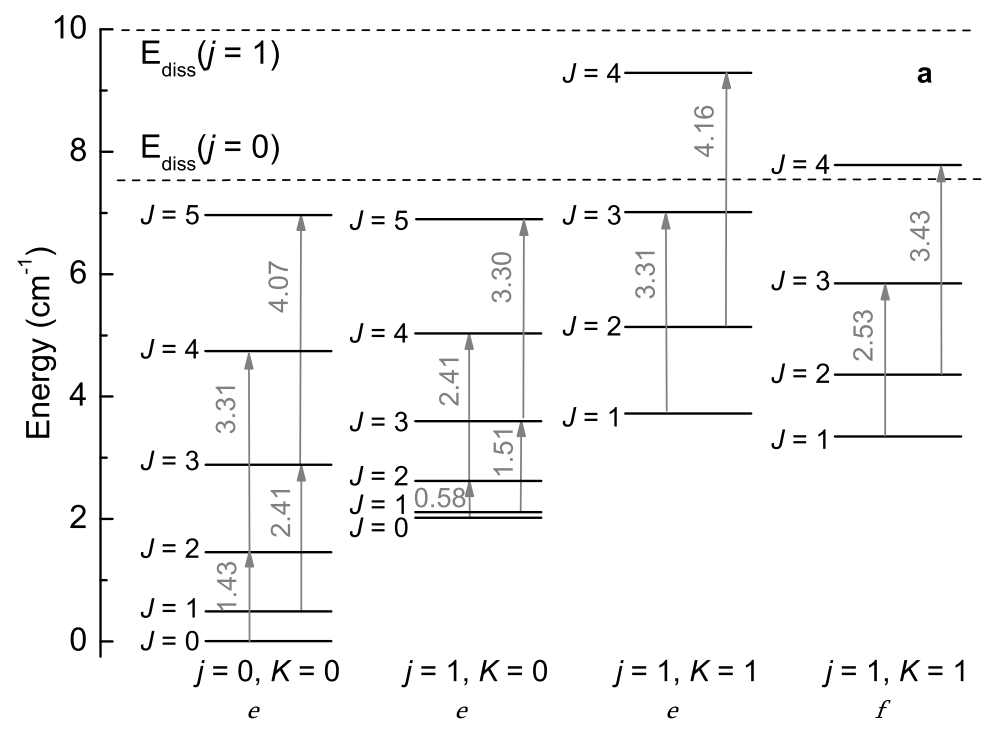

Fig. 8 Rotational energy level diagrams of $\mathrm{C}_{2} \mathrm{H}_{2}-\mathrm{He}$. The energy levels are grouped with respect to quantum numbers $j, K$ and parity labels $e, f$. The vertical and diagonal arrows designate the transitions observed experimentally. The numbers next to the arrows designate the corresponding transition frequencies in $\mathrm{cm}^{-1}$ (see table 2). The horizontal dashed lines indicate the dissociation limits corresponding to $\mathrm{C}_{2} \mathrm{H}_{2}$ in $j=0$ or $j=1$, respectively.

where measurements up to $600 \mathrm{ps}$ are possible, is not unreasonable. For example, the $\mathrm{CO}_{2}-\mathrm{He}$ complex has a lower limit of $6 \mathrm{~ns}$ for the predissociation life times, as determined by laser spectroscopy measurements ${ }^{103}$.

\subsection{Analysis of the rotational energy level pattern of $\mathrm{C}_{2} \mathrm{H}_{2}-\mathrm{He}$}

The computed level structure and the analysis of the wave functions shows that $\mathrm{C}_{2} \mathrm{H}_{2}-\mathrm{He}$ does not behave like a typical linear molecule in spite of its linear electronic minimum energy structure. Two limiting zero order models and corresponding labelling schemes can be used to describe the present situation, namely the rigid linear molecule picture in which $\mathrm{He}$ executes a bending motion, or the free internal rotor (potential-free) asymptote with a freely rotating $\mathrm{C}_{2} \mathrm{H}_{2}$ unit. The numerically exact wave functions obtained for given rigorous quantum numbers $J$ and $p$ in the present calculations can be projected onto either zero order representation in order to assign corresponding approximate quantum numbers and an additional analysis using an adiabatic projection is possible.

The bending vibration of linear molecules is two-dimensional and described by the quantum label $v_{\text {lin }}^{\ell}$, where the quantum number $\ell$ of the vibrational angular momentum $\hat{\ell}$ is given by $\ell=v_{\operatorname{lin}}, v_{\operatorname{lin}}-2, \cdots,-v_{\operatorname{lin}}$ leading to $v_{\operatorname{lin}}+1$ fold degeneracy of the level $v_{\text {lin }}$. Due to angular momentum conservation, $\hat{\ell}_{z}=\hat{J}_{z}$ also holds for linear triatomic molecules, where $\hat{\mathbf{J}}$ is the total rotation. This means that the first bending level for $J=0$ is $2 v_{\text {lin }}^{0}$ and that $v_{\text {lin }}^{1}$ is accessible only for $J \geq 1$. Above the angular barrier, the bending energy pattern shows the onset of 
a two-dimensional rotor structure. The latter structure arises in the potential-free situation and is characterised by $\ell=v_{\text {lin }}, v_{\text {lin }}-1, \cdots,-v_{\text {lin }}$, such that the degeneracy of the level $v_{\operatorname{lin}}$ is $2 v_{\operatorname{lin}}+1$. In the free-rotor limit, Coriolis interaction may prominently affect the overall molecular behaviour. ${ }^{104}$

A very useful quantitative analysis of the exact wavefunctions is possible through an adiabatic projection scheme designed for the DVR-DGB approach in the spirit of the method previously developed for tetratomic molecules. ${ }^{105}$ The radial eigenvalues computed for each angular DVR point during the construction of the full problem provide adiabatic angular profiles for a given stretching quantum number $v_{s}$. The lowest of these profiles corresponding to $v_{s}=0 \mathrm{de}-$ fines the adiabatic ground state bending profile ${ }^{\text {adi }} V^{0}(\theta)$, which is depicted in Fig. 9, together with the minimum energy path $V_{\mathrm{MEP}}(\theta)$ along the Jacobi angle $\theta$. The ${ }^{\text {adi }} V^{0}(\theta)$ profile differs from $V_{\text {MEP }}(\theta)$ by the angle dependent ground-state energy of the intermolecular stretching vibration and provides a more useful rationalisation of the angular motion of the complex. Fig. 9 shows that the inclusion of the ground-state stretching energy lowers the effective barrier height at $\theta=90^{\circ}$ from $8.3 \mathrm{~cm}^{-1}$ for $V_{\mathrm{MEP}}(\theta)$ to $3.4 \mathrm{~cm}^{-1}$ for ${ }^{\text {adi }} V^{0}(\theta)$ due to strong stretch-bend coupling. Another interesting observation is the very weak $\theta$ dependence of adi $V^{0}(\theta)$ for $\theta \in\left(0,45^{\circ}\right)$ and $\theta \in\left(135^{\circ}, 180^{\circ}\right)$, whereas $V_{\text {MEP }}(\theta)$ shows a somewhat parabolic $\theta$ shape in this region. Similar flat potentials over a wide angular range around linearity are typical for quasi-linear molecules ${ }^{106}$.

Solving the angular problem for an adiabatic profile with given $v_{s}$ provides bending functions with well defined adiabatic quantum numbers, $v_{s}$ and $v_{b}$. The functions $\left|v_{b}, v_{s} ; K\right\rangle$, where $K$ is the body-fixed $z$-projection of $\hat{\mathbf{J}}$, are used to construct adiabatic expansions of the exact wavefunctions, which allow assignments by identification of the dominant zero-order contribution(s). The adiabatic energies adi $\varepsilon^{(K)}$ differ from the exact rovibrational energies $E^{(J, p)}$ due to missing Coriolis coupling and bend-stretch coupling contributions.

The bound rovibrational states for $J=0$ and 1 in Fig. 9 are labelled by $\left(v_{b} ; K\right)$ using the adiabatic quantum number $v_{b}$ and the quantum number $K$. The complex has three bound $J=0$ states. As seen in Fig. 9, only the vibrational ground state falls below the adiabatic isomerisation barrier (saddle point in T-shaped arrangement) at ${ }^{\text {adi }} V^{0}\left(\theta=90^{\circ}\right)=-5.768 \mathrm{~cm}^{-1}$. The excited bending and stretching levels are 2.0 and $7.4 \mathrm{~cm}^{-1}$ above the ground state, respectively. Note that the stretching state at $-0.002 \mathrm{~cm}^{-1}$ is very marginally bound and further work will be necessary to establish the nature of this state. Fig. 9 provides additional state labels in terms of $[j, \ell ; K]$. This alternative scheme uses the angular momentum of $\mathrm{C}_{2} \mathrm{H}_{2}, \hat{\mathbf{j}}$, and the end-over-end angular momentum of the complex, $\hat{\ell}$. Then $\hat{\mathbf{J}}=\hat{\mathbf{j}}+\hat{\ell}$, whereas the parity is $(-1)^{j+\ell}$. For the $z$-axis along the Jacobi $\mathbf{R}$ vector (see Fig. 6), $\ell_{z}=0$ also holds. In terms of $j$ and $\ell$, the quantum label reads $[j, \ell ; K]$ for a given $J$ and parity $p$. The correlation between the labels $\left(v_{b} ; K\right)$ and $[j, \ell ; K]$ can be established by means of the angular momentum coupling rules and related Clebsch-Gordan coefficients. ${ }^{107}$ For $j=0$, we obtain $[0, J ; 0]$. For $j=1$, there are three possible level groups: $[1, J+1 ; 0]$ (e levels), $[1, J-1 ; 1]$ (e levels), and $[1, J ; 1]$ ( $f$ levels). For the parity labelling in terms of $e$ and $f$ states, see Ref. ${ }^{108}$.

The energy level structure in Fig. 9 shows several features which are indicative of an early onset of a free-rotor energy pattern. The $J=0$ energy of the

16 | Faraday Discuss., [year], [vol], 1-25

This journal is $\odot$ The Royal Society of Chemistry [year] 


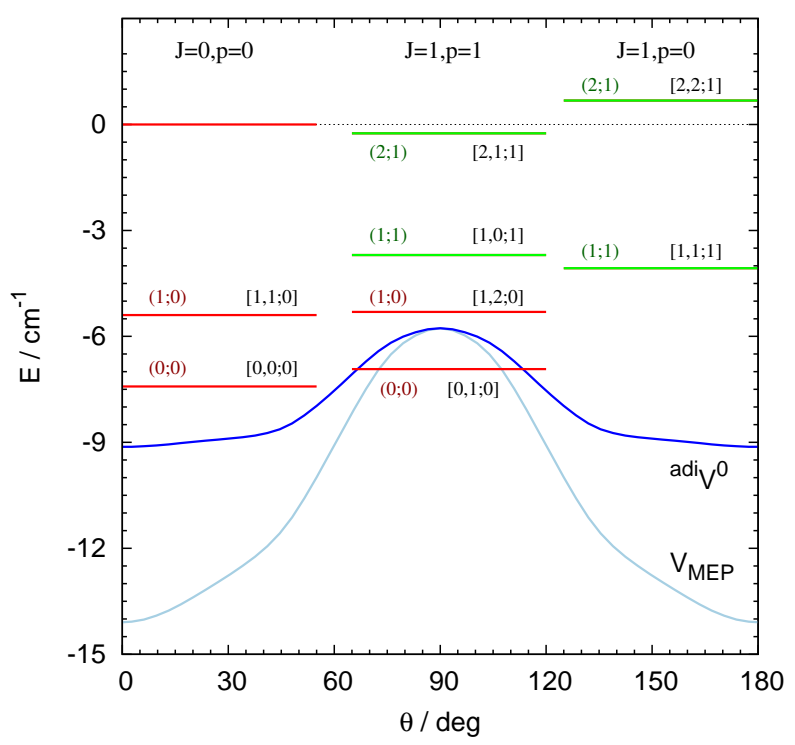

Fig. 9 Minimum energy path $V_{\text {MEP }}$ and effective ground-state potential ${ }^{\text {adi }} V^{0}$ along the Jacobi angle $\theta$. The curve $V_{\text {MEP }}$ is shifted upward by $10.99 \mathrm{~cm}^{-1}$ to coincide with ${ }^{\text {adi }} V^{0}$ at $\theta=90^{\circ}$. The quantum labels of the exact rovibrational states are given as both $\left(v_{b} ; K\right)$ and $[j, l ; K]$. The $J=0, p=0$ level without a quantum label is the excited stretching state. $K=0$ and $K=1$ levels are additionally coded in red and green, respectively.

bending vibration is below the energy of the complex rotating with $K=1$. This is contrary to the situation in ordinary linear molecules, where the first bending $J=0$ level, $2 v_{\text {lin }}^{0}$, is above the first $K=1$ level, $v_{\text {lin }}^{1}$. The splitting of the $K=1$ levels of $0.37 \mathrm{~cm}^{-1}$ for $J=1$ is large, in particular in relation to the very low energy scales in the present complex, and indicates a very strong rotation-vibration interaction. To clarify these points in more detail, we compare the exact energies $E^{(J, p)}$ with the adiabatic energies ${ }^{\text {adi }} \varepsilon^{(K)}$ for $J=1$ in Fig. 10. For $p=1$, the adiabatic $K=0$ and $K=1$ components are prominently pushed apart by Coriolis interaction. The global effect of this strong Coriolis coupling is clearly visible in Fig. 8a: without this coupling the $j=1, K=1 e$ and $f$ levels are degenerate. The levels of the $j=1, K=1 e$ stack are, however, systematically pushed upwards by Coriolis coupling with the $j=1, K=0 e$ levels of the same $J$, such that the latter are compressed downward. As a result the level energies in the $j=0, K=0$ stack increase more rapidly leading to a cross-over at $J=5$. Energy levels exhibiting Coriolis-type resonances are identified with the help of the probability ${ }^{(J)} P_{K}$ that the wave function takes a certain $K$ value. Coriolis mixing becomes more distinct as $v_{b}$ increases. This is seen through the increase of the separations between the resulting exact levels and the decrease of the ${ }^{(J)} P_{K}$ values with increasing $v_{b}$ in Fig. 10.

The effect of Coriolis coupling appears marginal for the lowest $J=1$ state in Fig. 10. This is consistent with Fig. 9, which shows that this level lies below the isomerisation barrier adi $V^{0}\left(\theta=90^{\circ}\right)$ for $J=0$ and $J=1$. The vibrational ground state for $J>2$ is, however, already above this barrier, such that it may also 


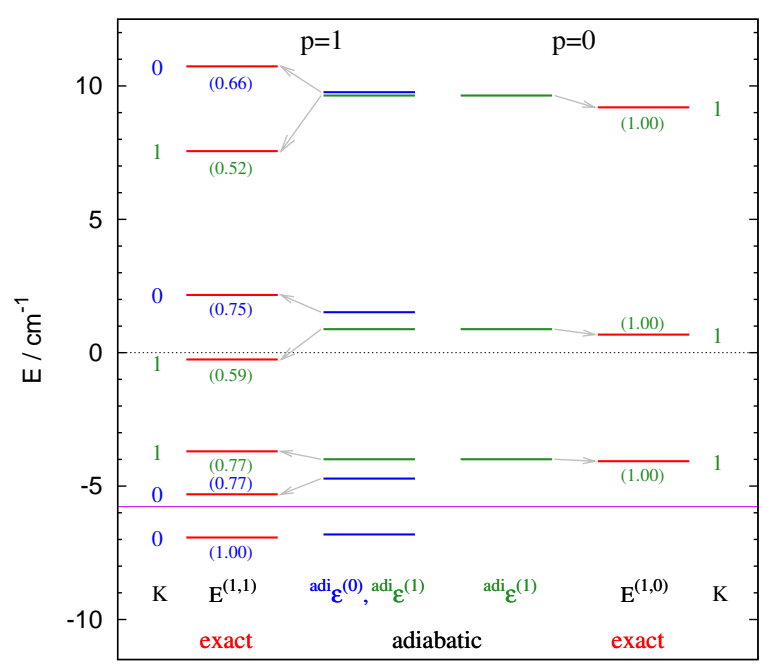

Fig. 10 Full-dimensional accurate level energies $E^{(J, p)}$ and adiabatic level energies ${ }^{\text {adi }} \varepsilon^{(K)}$ for $J=1$. The horizontal line at $-5.768 \mathrm{~cm}^{-1}$ indicates the position of the ground-state adiabatic isomerisation barrier (T-shape barrier). Arrows connect ${ }^{\text {adi }} \varepsilon^{(K)}$ levels with the exact eigenstates $E^{(J, p)}$ (red horizontal bars) originating from them. The quantity ${ }^{(J)} P_{K}$ is given in parentheses. Adiabatic $K=0$ and $K=1$ levels are additionally coded in blue and green, respectively.

experience Coriolis mixing. This perturbation became visible when we fitted the rotational excitation of the vibrational ground state with the two-parameter and three-parameter effective term formulas

$$
\begin{aligned}
{ }^{(2)} E_{0}(J)= & { }^{(2)} B_{0} J(J+1)-{ }^{(2)} D_{0} J^{2}(J+1)^{2}, \\
{ }^{(3)} E_{0}(J)= & { }^{(3)} B_{0} J(J+1)-{ }^{(3)} D_{0} J^{2}(J+1)^{2} \\
& +{ }^{(3)} H_{0} J^{3}(J+1)^{3} .
\end{aligned}
$$

Including rotational transitions up to $J=5$, we obtain $\left[{ }^{(2)} B_{0},{ }^{(2)} D\right]=[0.2466,0.0005]$ and $\left[{ }^{(3)} B_{0},{ }^{(3)} D\right]=[0.2451,0.0003]$ with ${ }^{(3)} H=3 \cdot 10^{-6}$, where the standard deviation of the fit was $\sigma=3 \cdot 10^{-3}$ and $2 \cdot 10^{-4}$, respectively (all values given in $\mathrm{cm}^{-1}$ ). The sensitivity of $B_{0}$ on the number of fitting parameters and the large centrifugal $D$ constant are indications of a rotational perturbation affecting the vibrational ground state. This can be seen in Fig. 11, which shows the importance of the centrifugal distortion constant $D$ in the description of the rotational excitation in the vibrational ground state. The strong curvature seen for the levels $[1 ; 0]^{e}$ (black line) and $[1 ; 1]^{e}$ (blue line) results from Coriolis coupling between these two states.

In the free-rotor limit (potential-free situation), the level energy is approximated by

$$
E_{j, \ell}=B \ell(\ell+1)+b j(j+1),
$$

where $B$ and $b$ are the rotational constants of the complex and the monomer, respectively, and $b>B$. In this limit the bending $[1,1 ; 0]$ state and the $K=1$ level 


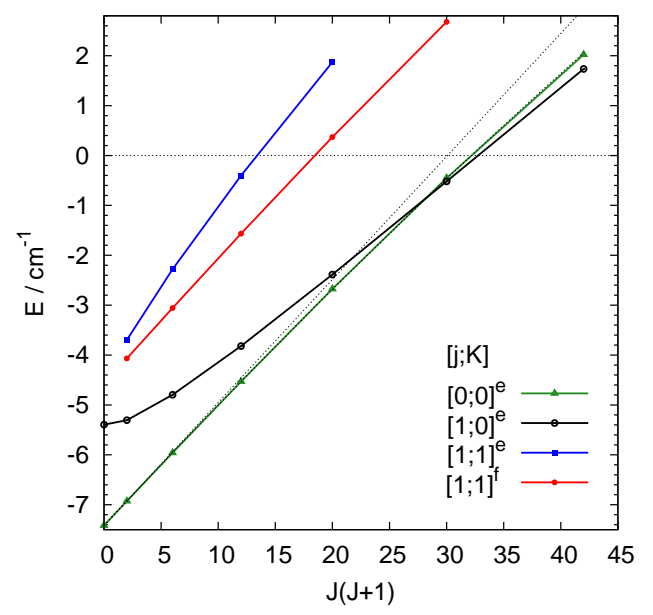

Fig. 11 Rotational energy as a function of $J(J+1)$. The dotted line stands for $E_{0}={ }^{(2)} B_{0} J(J+1)$.

$[1,1 ; 1]^{f}$ would have the same energy of $2(B+b)$. The $K=1$ level $[1,0 ; 1]^{e}$ is then higher in energy by $2 b$ than the ground vibrational state $[0,0 ; 0]$. The splitting of the $K=1$ levels $[1,0 ; 1]^{e}$ and $[1,1 ; 1]^{f}$ would be $2 B$, with the $f$ state above the $e$ state. In qualitative terms, the rovibrational structure of Fig. 9 features some of these properties. In quantitative terms, however, there are differences arising primarily from the very prominent Coriolis rotation-vibration interaction, such as the ordering of the $e$ and $f$ states with $K=1$, which is reversed with respect to the potential-free situation.

\section{Conclusions}

In conclusion, we have applied impulsive alignment to build rotational wavepackets in complexes between acetylene and helium atoms. The phase of the wavepacket, represented by the degree of alignment, was measured as a function of time using Coulomb explosion. A Fourier transform revealed a rich spectrum of transitions between bound rovibrational eigenstates of the $\mathrm{C}_{2} \mathrm{H}_{2}-\mathrm{He}$ complex, which is in excellent agreement with theoretical predictions. The experimental verification of essentially all rotational eigenstates up to the dissociation threshold of the complex substantiated a detailed theoretical assessment of the level structure. Providing access to a large set of rotational states even for molecular systems with near zero dipole moment, as exemplified by the $\mathrm{C}_{2} \mathrm{H}_{2}-\mathrm{He}$ complex, is a particular strength of the impulsive alignment technique.

The $\mathrm{C}_{2} \mathrm{H}_{2}-\mathrm{He}$ complex is a highly delocalised system. This is the result of the small mass of helium in combination with a small angular barrier on the potential energy surface. The effective angular barrier is lowered further due to strong stretch-bend coupling, such that only two rovibrational eigenstates are below it. All states have a high probability in the barrier region. States above the barrier experience strong Coriolis coupling. In this respect the $\mathrm{C}_{2} \mathrm{H}_{2}-\mathrm{He}$ complex differs 
from linear complexes with a typical linear-molecule rovibrational structure, such as $\mathrm{HCN}-\mathrm{Ar}^{102}$ and T-shaped complexes with a slightly asymmetric prolate top structure such as $\mathrm{CO}_{2}-\mathrm{He}^{103,109}$ and $\mathrm{C}_{2} \mathrm{H}_{2}^{+}-\mathrm{Ar}^{110}$ whose rotational levels can be well described in terms of effective rotational parameters. The present system exhibits some similarities with the complex $\mathrm{CO}^{+}-\mathrm{He},{ }^{111}$ which was found to exhibit an early change of rovibrational patterns due to a very low barrier to linearity.

These findings enable the extension of the impulsive alignment method to other molecular complexes and to clusters comprising multiple atoms. In the case of molecule-helium complexes by increasing the number of helium atoms it will be possible to gain new insight into incipient superfluid effects. Because the impulsive alignment method does not require the molecule to possess a permanent electric dipole moment it can be applied to a wide range of molecules, including homonuclear diatomics and homonuclear atomic clusters. Incipient superfluid effects for these types of systems have not been explored previously. This methodology also offers the prospect of studying small rare gas clusters or homonuclear metal clusters, testing van der Waals forces and chemical interactions, respectively.

Provided the detection of alignment is successful for these systems impulsive alignment should then decisively facilitate the spectral analysis through the control of the constitution of the wavepacket by adjusting the pump laser power, making it possible to access information on rotational levels at higher energies. Equivalently, for low pump powers it is possible to selectively excite transitions from the lowest quantum states, thereby facilitating the interpretation of congested spectral data. All of this is possible in combination with a very cold molecular beam, enabling the formation of weakly bound species and an initial population of only the lowest quantum levels.

\section{Acknowledgements}

The authors wish to thank STFC for access to the Artemis facility at the Rutherford Appleton Laboratory and the University of Leicester for funding to support the studentship for GG associated with this project. We would like to thank S. Hook, P. Rice, N. Rodrigues and S. Thornton for technical support during the experiment. KvH kindly acknowledges funding by STFC (seed corn fund for experiments using 4th generation light sources) and the Leverhulme Trust (F/00212/AH). LGML acknowledges financial support from the Mexican Consejo Nacional de Ciencia y Tecnología (CONACYT) Scholarship number 310668, ID 215334. RSM would like to thank the Royal Society for a University Research Fellowship (UF100047), ML and MM acknowledge computational resources funded through ANR grant ANR-08-BLAN-0146-01. KHMB and JT acknowledge financial support by the Deutsche Forschungsgemeinschaft through SFB 652.

\section{References}

1 T. Seideman, J. Chem. Phys., 1995, 103, 7887-7896. 
2 B. Friedrich and D. Herschbach, Phys. Rev. Lett., 1995, 74, 4623-4626.

3 J. J. Larsen, H. Sakai, C. P. Safvan, I. Wendt-Larsen and H. Stapelfeldt, J. Phys. Chem., 1999, 111, 7774-7781.

4 F. Rosca-Pruna and M. J. J. Vrakking, Phys. Rev. Lett., 2001, 87, 153902.

5 M. Tsubouchi, B. J. Whitaker, L. Wang, H. Kohguchi and T. Suzuki, Phys. Rev. Lett., 2001, 86, 4500.

6 H. Stapelfeldt and T. Seideman, Rev. Mod. Phys., 2003, 75, 543-557.

7 T. Seideman and E. Hamilton, Adv. At., Mol., Opt. Phys., 2006, 52, 289-329.

8 C. Vallance, Phys. Chem. Chem. Phys., 2011, 13, 14427-14441.

9 M. Machholm and N. Henriksen, Phys. Rev. Lett., 2001, 87, 193001.

10 J. P. Heritage, T. K. Gustafson and C. H. Lin, Phys. Rev. Lett., 1975, 34, 1299-1302.

11 P. M. Felker, J. Chem. Phys., 1992, 96, 7844-7857.

12 M. Morgen, W. Price, L. Hunziker, P. Ludowise, M. Blackwell and Y. Chen, Chem. Phys. Lett., 1993, 209, 1-9.

13 T. S. Rose, W. L. Wilson, G. Wäckerle and M. D. Fayer, J. Chem. Phys., 1987, 86, 5370-5391.

14 V. V. Matylitsky, W. Jarzeba, C. Riehn and B. Brutschy, J. Raman Spectr, 2002, 33, 877-883.

15 P. W. Joireman, L. L. Connell, S. M. Ohline and P. M. Felker, J. Phys. Chem., 1991, 95, 4935-4939.

16 V. A. Venturo, P. M. Maxton and P. M. Felker, J. Phys. Chem., 1992, 96, 5234-5237.

17 V. A. Venturo, P. M. Maxton, B. F. Henson and P. M. Felker, J. Chem. Phys., 1992, 96, 7855-7858.

18 M. Cho, M. Du, N. F. Scherer, G. R. Fleming and S. Mukamel, J. Chem. Phys., 1993, 99, 2410-2428.

19 P. W. Dooley, I. V. Litvinyuk, K. F. Lee, D. M. Rayner, M. Spanner, D. M. Villeneuve and P. B. Corkum, Phys. Rev. A, 2003, 68, 023406.

20 V. Renard, M. Renard, S. Guérin, Y. T. Pashayan, B. Lavorel, O. Faucher and H.-R. Jauslin, Phys. Rev. Lett., 2003, 90, 153601.

21 E. Hamilton, T. Seideman, T. Ejdrup, M. D. Poulsen, C. Z. Bisgaard, S. S. Viftrup and H. Stapelfeldt, Phys. Rev. A, 2005, 72, 043402.

22 E. Péronne, M. D. Poulsen, C. Z. Bisgaard, H. Stapelfeldt and T. Seideman, Phys. Rev. Lett., 2003, 91, 043003.

23 J. G. Underwood, B. J. Sussman and A. Stolow, Phys. Rev. Lett., 2005, 94, 143002.

24 K. F. Lee, D. M. Villeneuve, P. B. Corkum, A. Stolow and J. G. Underwood, Phys. Rev. Lett., 2006, 97, 173001.

25 A. Rouzée, E. Hertz, B. Lavorel and O. Faucher, J. Phys. B, 2008, 41, 074002.

26 V. Kumarappan, L. Holmegaard, C. Martiny, C. B. Madsen, T. K. Kjeldsen, S. S. Viftrup, L. B. Madsen and H. Stapelfeldt, Phys. Rev. Lett., 2008, 100, 093006-093006.

27 L. Holmegaard, J. L. Hansen, L. Kalhøj, S. L. Kragh, H. Stapelfeldt, F. Filsinger, J. Küpper, G. Meijer, D. Dimitrovski, M. Abu-Samha et al.,

This journal is $\odot$ The Royal Society of Chemistry [year]

Faraday Discuss., [year], [vol], 1-25 | 21 
Nature Phys., 2010, 6, 428-432.

28 J. Itatani, J. Levesque, D. Zeidler, H. Niikura, H. Pépin, J.-C. Kieffer, P. B. Corkum and D. M. Villeneuve, Nature, 2004, 432, 867-871.

29 K. Miyazaki, M. Kaku, G. Miyaji, A. Abdurrouf and F. H. M. Faisal, Phys. Rev. Lett., 2005, 95, 243903.

30 T. Kanai, S. Minemoto and H. Sakai, Nature, 2005, 435, 470-474.

31 M. Meckel, D. Comtois, D. Zeidler, A. Staudte, D. Pavičić, H. C. Bandulet, H. Pépin, J. C. Kieffer, R. Dörner, D. M. Villeneuve and P. B. Corkum, Science, 2008, 320, 1478-1481.

32 J. Küpper, S. Stern, L. Holmegaard, F. Filsinger, A. Rouzée, A. Rudenko, P. Johnsson, A. V. Martin, M. Adolph, A. Aquila, S. c. v. Bajt, A. Barty, C. Bostedt, J. Bozek, C. Caleman, R. Coffee, N. Coppola, T. Delmas, S. Epp, B. Erk, L. Foucar, T. Gorkhover, L. Gumprecht, A. Hartmann, R. Hartmann, G. Hauser, P. Holl, A. Hömke, N. Kimmel, F. Krasniqi, K.-U. Kühnel, J. Maurer, M. Messerschmidt, R. Moshammer, C. Reich, B. Rudek, R. Santra, I. Schlichting, C. Schmidt, S. Schorb, J. Schulz, H. Soltau, H. Spence, John C. D. Starodub, L. Strüder, J. Thøgersen, J. Vrakking, Marc J. G. Weidenspointner, T. A. White, C. Wunderer, G. Meijer, J. Ullrich, H. Stapelfeldt, D. Rolles and H. N. Chapman, Phys. Rev. Lett., 2014, 112, 083002.

33 J.-M. Hartmann, C. Boulet, T. Vieillard, F. Chaussard, F. Billard, O. Faucher and B. Lavorel, J. Chem. Phys., 2013, 139, 024306.

34 W. A. de Heer, Rev. Mod. Phys., 1993, 65, 611-676.

35 T. P. Martin, Phys. Rep., 1996, 273, 199-241.

36 F. Baletto and R. Ferrando, Rev. Mod. Phys., 2005, 77, 371.

37 E. Roduner, Chem. Society Rev.s, 2006, 35, 583-592.

38 Y. Xia, Y. Xiong, B. Lim and S. Skrabalak, Angew. Chem. Int. Ed., 2008, 48, 60-103.

39 D. V. Talapin, J.-S. Lee, M. V. Kovalenko and E. V. Shevchenko, Chem. Rev., 2009, 110, 389-458.

40 E. Becker, Laser and Particle Beams, 1989, 7, 743-753.

41 R. Campargue, Atomic and Molecular Beams: The State of the Art 2000, Springer, 2001.

42 J. H. Parks, S. Pollack and W. Hill, J. Chem. Phys., 1994, 101, 6666-6685.

43 M. Maier-Borst, D. B. Cameron, M. Rokni and J. H. Parks, Phys. Rev. A, 1999, 59, R3162.

44 J. W. Hager, Rapid Commun. Mass. Spectrom., 2002, 16, 512-526.

45 J. T. Lau, J. Rittmann, V. Zamudio-Bayer, M. Vogel, K. Hirsch, P. Klar, F. Lofink, T. Möller and B. v. Issendorff, Phys. Rev. Lett., 2008, 101, 153401.

46 J. Higgins, C. Callegari, J. Reho, F. Stienkemeier, W. Ernst, K. Lehmann, M. Gutowski and G. Scoles, Science, 1996, 273, 629.

47 U. Buck and F. Huisken, Chem. Rev., 2000, 100, 3863-3890.

48 F. Stienkemeier, J. Higgins, W. E. Ernst and G. Scoles, Phys. Rev. Lett., 1995, 74, 3592-3595.

49 K. von Haeften, T. Laarmann, H. Wabnitz, T. Möller and K. Fink, J. Phys.

22 | Faraday Discuss., [year], [vol], 1-25

This journal is $\odot$ The Royal Society of Chemistry [year] 
Chem. A, 2011, 25, 7316-7326.

50 O. Echt, K. Sattler and E. Recknagel, Phys. Rev. Lett., 1981, 47, 1121-1124.

51 W. Knight, K. Clemenger, W. de Heer, W. Saunders, M. Chou and M. Cohen, Phys. Rev. Lett., 1984, 52, 2141-2143.

52 H. W. Kroto, J. R. Heath, S. C. O’Brien, R. F. Curl and R. E. Smalley, Nature, 1985, 318, 162-163.

53 W. A. de Heer, P. Milani and A. Chatelain, Phys. Rev. Lett., 1990, 65, 488.

54 J. Bucher, D. Douglass and L. Bloomfield, Phys. Rev. Lett., 1991, 66, 3052.

55 M. B. Knickelbein, Phys. Rev. Lett., 2001, 86, 5255-5257.

56 V. Kresin, Phys. Rev. B, 1989, 39, 3042.

57 R. Moro, R. Rabinovitch, C. Xia and V. V. Kresin, Phys. Rev. Lett., 2006, 97, 123401.

58 X. Xu, S. Yin, R. Moro, A. Liang, J. Bowlan and W. A. de Heer, Phys. Rev. $B, 2007,75,085429$.

59 J. Farges, M. De Feraudy, B. Raoult and G. Torchet, J. Chem. Phys., 1986, 84, 3491.

60 S. Krückeberg, D. Schooss, M. Maier-Borst and J. H. Parks, Phys. Rev. Lett., 2000, 85, 4494.

61 M. N. Blom, D. Schooss, J. Stairs and M. M. Kappes, J. Chem. Phys., 2006, 124, 244308.

62 F. Luo, C. F. Giese and W. R. Gentry, J. Chem. Phys., 1996, 104, 1151-1154.

63 B. S. Zhao, G. Meijer and W. Schöllkopf, Science, 2011, 331, 892-894.

64 C. Bostedt, E. Eremina, D. Rupp, M. Adolph, H. Thomas, M. Hoener, A. R. B. de Castro, J. Tiggesbäumker, K.-H. Meiwes-Broer, T. Laarmann, H. Wabnitz, E. Plönjes, R. Treusch, J. R. Schneider and T. Möller, Phys. Rev. Lett., 2012, 108, 093401.

65 T. Gorkhover, M. Adolph, D. Rupp, S. Schorb, S. Epp, B. Erk, L. Foucar, R. Hartmann, N. Kimmel, K.-U. Kühnel et al., Phys. Rev. Lett., 2012, 108, 245005.

66 Z. Bačić and R. E. Miller, J. Phys. Chem., 1996, 100, 12945-12959.

67 D. J. Nesbitt and R. Naaman, J. Chem. Phys., 1989, 91, 3801.

68 J. P. Toennies and A. F. Vilesov, Ann. Rev. Phys. Chem., 1998, 49, 1-41.

69 F. Stienkemeier and A. F. Vilesov, J. Chem. Phys., 2001, 115, 10119.

70 B. S. Dumesh and L. A. Surin, Phys.-Uspekhi, 2006, 49, 1113-1129.

71 F. Stienkemeier and K. K. Lehmann, J. Phys. B: At. Mol. Opt. Phys., 2006, 39, R127-R166.

72 J. P. Toennies and A. F. Vilesov, Angew. Chem. Int. Ed., 2004, 43, 26222648.

73 J. Küpper and J. Merritt, Int. Rev. Phys. Chem., 2007, 26, 249-287.

74 M. Barranco, R. Guardiola, S. Hernandez, R. Mayol, J. Navarro and M. Pi, J. Low Temp. Phys., 2006, 142, 1.

75 R. Moszynski, P. E. S. Wormer and A. van der Avoird, J. Chem. Phys., 1995, 102, 8385.

76 C. R. Munteanu and B. Fernández, J. Chem. Phys., 2005, 123, 014309.

77 M. Rezaei, N. Moazzen-Ahmadi, A. R. W. McKellar, B. Fernández and D. Farrelly, Mol. Phys., 2012, 110, 2743-2750. 
78 B. Fernández, C. Henriksen and D. Farrelly, Mol. Phys., 2013, 111, 1173.

79 D. Pentlehner, J. H. Nielsen, A. Slenczka, K. Mølmer and H. Stapelfeldt, Phys. Rev. Lett., 2013, 110, 093002.

80 U. Even, J. Jortner, D. Noy, N. Lavie and C. Cossart-Magos, J. Chem. Phys., 2000, 112, 8068.

81 A. Eppink and D. Parker, Rev. Sci. Instrum., 1997, 68, 3477.

82 O. Ghafur, A. Rouzée, A. Gijsbertsen, W. K. Siu, S. Stolte and M. J. J. Vrakking, Nature Phys., 2009, 5, 289-293.

83 G. Roberts, J. Nixon, J. Lecointre, E. Wrede and J. Verlet, Rev. Sci. Instr., 2009, 80, 053104.

84 V. Dribinski, A. Ossadtchi, V. A. Mandelshtam and H. Reisler, Rev. Sci. Instr., 2002, 73, 2634-2642.

85 G. Garcia, L. Nahon and I. Powis, Rev. Sci. Instr., 2004, 75, 4989.

86 A. Mikaberidze, U. Saalmann and J. M. Rost, Phys. Rev. Lett., 2009, 102, 128102 .

87 A. Przystawik, A. Kickermann, A. Al-Shemmary, S. Düsterer, A. M. Ellis, K. von Haeften, M. Harmand, S. Ramakrishna, H. Redlin, L. Schroedter, M. Schulz, T. Seideman, N. Stojanovic, J. Szekely, F. Tavella, S. Toleikis and T. Laarmann, Phys. Rev. A, 2012, 85, 052503.

88 M. Herman, A. Campargue, M. I. El Idrissi and J. Van der Auwera, J. Phys. Chem. Ref. Data, 2003, 32, 922-1361.

89 G. Herzberg, Molecular Spectra and Molecular Structure. Volume II: Infrared and Raman Spectra of Polyatomic Molecules, D. Van Nostrand, 16th edn., 1945.

90 L. Surin, A. Potapov, B. Dumesh, S. Schlemmer, Y. Xu, P. Raston and W. Jäger, Phys. Rev. Lett., 2008, 101, 233401.

91 J. Tang, Y. Xu, A. R. W. McKellar and W. Jäger, Science, 2002, 297, 20302033.

92 M. Hartmann, R. E. Miller, J. P. Toennies and A. F. Vilesov, Phys. Rev. Lett., 1995, 75, 1566.

93 T. H. Dunning, Jr., J. Chem. Phys., 1989, 90, 1007.

94 R. A. Kendall, T. H. Dunning, Jr. and R. J. Harrison, J. Chem. Phys., 1992, 96, 6796.

95 H.-J. Werner, P. J. Knowles, G. Knizia, F. R. Manby, M. Schütz et al., MOLPRO, version 2012.1, a package of ab initio programs, 2012, see http://www.molpro.net.

96 K. A. Peterson, D. E. Woon and T. H. Dunning Jr., J. Chem. Phys., 1994, 100, 7410.

97 K. A. Peterson and G. C. McBane, J. Chem. Phys., 2005, 123, 084314.

98 T. Helgaker, W. Klopper, H. Koch and J. Noga, J. Chem. Phys., 1997, 106, 9639-9646.

99 B. Fernández, C. Henriksen and D. Farrelly, Mol. Phys., 2013, 111, $1173-$ 1177.

100 K. T. Tang and J. P. Toennies, J. Chem. Phys., 1984, 80, 3726.

101 W. H. Press, S. A. Teukolsky, W. T. Vetterling and B. P. Flannery, Numerical Recipes in FORTRAN. The Art of Scientific Computing, 2nd Ed., Cambridge

24 | Faraday Discuss., [year], [vol], 1-25

This journal is @ The Royal Society of Chemistry [year] 
University Press, Cambridge, 1992.

102 M. Mladenović and Z. Bačić, J. Chem. Phys., 1991, 94, 4988.

103 M. J. Weida, J. M. Sperhac, D. J. Nesbitt and J. M. Hutson, J. Chem. Phys., 1994, 101, 8351.

104 M. Mladenović, P. Botschwina, P. Sebald and S. Carter, Theo. Chem. Acc., 1998, 100, 134.

105 M. Mladenović, Spectrochim. Acta, Part A, 2002, 58, 795.

106 B. P. Winnewisser, Molecular Spectroscopy: Modern Research, Academic Press, Orlando, 1985, vol. 3.

107 R. N. Zare, Angular Momentum, J. Wiley \& Sons, New York, 1988.

108 M. Brown, J. T. Hougen, K.-P. Huber, J. W. C. Johns, I. Kopp, H. LefebvreBrion, A. J. Merer, D. A. Ramsay, J. Rostas and R. N. Zare, J. Mol. Spectrosc., 1975, 55, 500-504.

109 Y. J. Xu and W. Jäger, 2001, 599, 211-217.

110 O. Dopfer, R. V. Olkhov, M. Mladenović and P. Botschwina, J. Chem. Phys., 2004, 121, 1744.

111 M. Mladenović and M. Lewerenz, Mol. Phys., 2013, 111, 2068-2085. 